\title{
Erratum to: Analyzing restricted fragments of the theory of linear arithmetic
}

\author{
Piotr Wojciechowski $^{1}$ - Pavlos Eirinakis ${ }^{2}$ (D) . \\ K. Subramani ${ }^{1}$
}

Published online: 3 March 2017

(C) Springer International Publishing Switzerland 2017

\section{Erratum to: Ann Math Artif Intell (2017) 79:245-266}

\section{DOI 10.1007/s10472-016-9525-7}

Owing to an error in the production process, the following article has been published incorrectly online. The article including the illustrations is presented hereafter.

\begin{abstract}
The theory of linear arithmetic (TLA), also known as the Theory of Rationals, is an extremely well-studied theory. It has been widely applied to a number of domains, including program verification and constraint specification. This paper discusses the computational complexities of two broad fragments of TLA, namely Quantified Linear Programs (QLPs) and Quantified Linear Implications (QLIs). These fragments are ideal for expressing specifications in real-time scheduling, and for modeling reactive systems. In this paper, we study the computational complexities of several variants of QLPs and QLIs. Our principal result shows that there exists a one-to-one correspondence between alternations in a class of
\end{abstract}

This research was supported in part by the National Science Foundation through Award CCF-1305054.

This work was supported by the Air Force Research Laboratory under US Air Force contract FA8750-16-3-6003. The views expressed are those of the authors and do not reflect the official policy or position of the Department of Defense or the U.S. Government.

The online version of the original articles can be found at http://dx.doi.org/10.1007/s10472-016-9525-7.

Pavlos Eirinakis

peir@aueb.gr

Piotr Wojciechowski

pwojciec@mix.wvu.edu

K. Subramani

k.subramani@mail.wvu.edu

1 LDCSEE, West Virginia University, Morgantown, WV 26506, USA

2 DMST, Athens University of Economics and Business, 10434 Athens, Greece 
QLIs and the complexity classes comprising the polynomial hierarchy $\mathbf{P H}$. In other words, for each class of the $\mathbf{P H}$, there exists a class of QLIs that is complete for that class. Our work mirrors the work in [L.J. Stockmeyer, The Polynomial-Time Hierarchy, Theoretical Computer Science, 3:1-22, 1977] which established the connection between the classes of PH and quantifier alternations in Quantified Boolean Formulas. Our results are surprising, since the variables in the fragments that we consider are continuous, as opposed to discrete.

Keywords Quantified linear programming · Quantified linear implication · Theory of real numbers with addition $\cdot$ Complexity classes

Mathematics Subject Classification (2010) 68Q17 $\cdot 03$ D15 $\cdot 90 \mathrm{C} 05$

\section{Introduction}

The Theory of Linear Arithmetic (TLA), also known as the Theory of Rationals (see Appendix A.1), has been well-studied in the literature, on account of its wide applicability to a number of practical domains. Indeed, this theory forms the backbone of most automated program verifiers [4]. This paper studies the computational complexities of some interesting fragments of TLA. In particular, it deals with Quantified Linear Programs (QLPs) and Quantified Linear Implications (QLIs). Quantified linear programming is the problem of checking whether a linear system is satisfiable with respect to a given quantifier string. The quantifier string specifies the sort of each variable (existential or universal) and the order in which they occur. Hence, quantified linear programming is a (non-trivial) generalization of linear programming. For each universally quantified variable, the quantifier string specifies a non-empty, continuous and closed interval on the real-line; this interval corresponds to the permissible values for the variable [33]. It is not hard to see that QLPs represent the conjunctive fragment of TLA with one major exception, viz., the universally quantified variables are bounded. Note that the bounds can be removed by rewriting the constraints implied by the QLP as an implication. The bounds on the universally quantified variables are placed in the Left Hand Side (LHS) of the implication, while the original constraints form the Right Hand Side (RHS). This is demonstrated in Example 1.

Example 1 Let us consider QLP (1):

$$
\forall y_{1} \in[0,1] \exists x_{1} \quad x_{1}+y_{1} \leq 1 .
$$

This is equivalent to QLI (2):

$$
\forall y_{1} \exists x_{1} \quad\left[0 \leq y_{1} \leq 1 \rightarrow x_{1}+y_{1} \leq 1\right] .
$$

Allowing an arbitrary linear system on the LHS of the implication results in a Quantified Linear Implication (QLI) [14]. Note that the abbreviations QLP and QLI and their variants PQLP, UQLP, PQLI and UQLI are used to denote both a class of problems and specific instances (see Section 2). The usage is clarified by the context.

In this paper, we examine some new variants of QLP and QLI, and establish their computational complexities. We also settle several open questions in the literature regarding QLI. We prove that the variants of QLP and QLI in which every universally quantified variable is partially bounded can be decided in polynomial time. Similarly, we show that the variants of these problems in which every universally quantified variable is unbounded can be decided in polynomial time. We also show that corresponding to each class of the polynomial hierarchy 
(PH) [27, 28], there exists some instantiation of the QLI framework that is complete for this class. This is interesting due to the fact that QLI covers the PH using only continuous variables, thus providing a continuous analogue to the results of [32]. Finally, we examine the computational complexities of some classes of QLI with one quantifier alternation.

The rest of this paper is organized as follows: Section 2 formally describes the problems that we consider in this paper. Section 3 describes the motivation for our work. In Section 4, we survey related results in the literature. Sections 5 and 6 discuss variants of QLP and QLI respectively from the perspective of computational complexity. Section 7 proves that Quantified Linear Implication covers the polynomial hierarchy. Related complexity results are described in Section 8. We conclude in Section 9 by summarizing our contributions and outlining avenues for future research.

\section{Statement of problems}

We use the standard notation of linear algebra [29] to formally present the basic notions of this paper. $\mathbb{R}$ is the set of real numbers. Let capital bold letters $(\mathbf{A}, \mathbf{B}, \ldots)$ denote matrices and small bold letters $(\mathbf{x}, \mathbf{y}, \mathbf{b}, \ldots)$ denote column vectors. $\mathbf{0}$ is the column vector with all elements equal to 0 . The product of $\mathbf{A}$ and $\mathbf{x}$ is denoted by $\mathbf{A} \cdot \mathbf{x}$. Likewise, let $\mathbf{x} \cdot \mathbf{y}$ denote the inner product of $\mathbf{x}$ and $\mathbf{y}$. Finally, we assume that the dimensions of vectors and matrices in products are compatible.

A QLP is a conjunction of linear constraints in which every variable is either existentially or universally quantified:

$$
\exists \mathbf{x}_{1} \forall \mathbf{y}_{1} \in\left[\mathbf{l}_{1}, \mathbf{u}_{1}\right] \ldots \exists \mathbf{x}_{n} \forall \mathbf{y}_{n} \in\left[\mathbf{l}_{n}, \mathbf{u}_{n}\right] \mathbf{A} \cdot \mathbf{x}+\mathbf{N} \cdot \mathbf{y} \leq \mathbf{b} .
$$

In QLP (3),

(i) $\mathbf{x}_{1} \ldots \mathbf{x}_{n}$ is a partition of $\mathbf{x}$ with $\mathbf{x}_{1}$ possibly empty.

(ii) $\mathbf{y}_{1} \ldots \mathbf{y}_{n}$ is a partition of $\mathbf{y}$ with $\mathbf{y}_{n}$ possibly empty.

(iii) $\mathbf{l}_{i}$ is the lower bound for $\mathbf{y}_{i}, i=1, \ldots, n$.

(iv) $\mathbf{u}_{i}$ is the upper bound for $\mathbf{y}_{i}, i=1, \ldots, n$.

Note that in a QLP every universally quantified variable is bounded from above and below. It is clear that a QLP that in which every variable is existentially quantified is a Linear Program (LP).

We now introduce two variants of Quantified Linear Programming, both of which alter the nature of the bounds on the universal variables.

A Partially-bounded Quantified Linear Program (PQLP) is a QLP in which every universally quantified variable is only bounded on one side.

$$
\exists \mathbf{x}_{1} \forall \mathbf{y}_{1} \in\left[\mathbf{l}_{1},+\infty\right) \ldots \exists \mathbf{x}_{n} \forall \mathbf{y}_{n} \in\left(-\infty, \mathbf{u}_{\mathrm{n}}\right] \mathbf{A} \cdot \mathbf{x}+\mathbf{N} \cdot \mathbf{y} \leq \mathbf{b}
$$

Without loss of generality, we can assume this single bound forces each such variable to be non-negative:

$$
\exists \mathbf{x}_{1} \forall \mathbf{y}_{1} \in[\mathbf{0},+\infty) \ldots \exists \mathbf{x}_{n} \forall \mathbf{y}_{n} \in[\mathbf{0},+\infty) \mathbf{A} \cdot \mathbf{x}+\mathbf{N} \cdot \mathbf{y} \leq \mathbf{b}
$$

This assumption holds because we can always transform any PQLP into the desired form. This is demonstrated in Example 2.

Example 2 Consider PQLP (4):

$$
\forall y_{1} \in(-\infty,-2] \exists x_{1} \quad x_{1}+y_{1} \leq 1 .
$$


We can change the upper bound on $y_{1}$ into a lower bound by negating all instances of $y_{1}$ in PQLP (4). This results in PQLP (5):

$$
\forall y_{1} \in[2,+\infty) \exists x_{1} \quad x_{1}-y_{1} \leq 1 .
$$

Then, we can change the lower bound on $y_{1}$ from 2 to 0 by replacing all instances of $y_{1}$ in PQLP (5) with $\left(y_{1}+2\right)$. This results in PQLP (6):

$$
\forall y_{1} \in[0,+\infty) \exists x_{1} \quad x_{1}-\left(y_{1}+2\right) \leq 1 .
$$

An Unbounded Quantified Linear Program (UQLP) is a QLP where there are no bounds on any universal variable:

$$
\exists \mathbf{x}_{1} \forall \mathbf{y}_{1} \ldots \exists \mathbf{x}_{n} \forall \mathbf{y}_{n} \mathbf{A} \cdot \mathbf{x}+\mathbf{N} \cdot \mathbf{y} \leq \mathbf{b}
$$

Note that UQLP represents the conjunctive fragment of TLA.

QLIs generalize the notion of polyhedral inclusion. The typical QLI has the following form:

$$
\exists \mathbf{x}_{1} \forall \mathbf{y}_{1} \ldots \exists \mathbf{x}_{n} \forall \mathbf{y}_{n} \quad[\mathbf{A} \cdot \mathbf{x}+\mathbf{N} \cdot \mathbf{y} \leq \mathbf{b} \rightarrow \mathbf{C} \cdot \mathbf{x}+\mathbf{M} \cdot \mathbf{y} \leq \mathbf{d}]
$$

In [14], a nomenclature was presented to represent the various classes of QLI. We briefly describe the nomenclature below:

Every QLI is exactly characterized by an ordered 3-tuple $\langle A, Q, T\rangle$. In this 3-tuple,

(i) $A$ denotes the number of quantifier alternations in the quantifier string.

(ii) $Q$ denotes the first quantifier.

(iii) $\quad T$ is an $(A+1)$-character string that specifies whether each quantified set of variables in the quantifier string appears on the Left, on the Right, or on Both sides of the implication.

Example 3 Consider the 3-tuple $\langle 1, \forall, \mathbf{B L}\rangle$. This indicates a problem described by QLI (8):

$$
\forall \mathbf{y} \exists \mathbf{x}[\mathbf{A} \cdot \mathbf{x}+\mathbf{N} \cdot \mathbf{y} \leq \mathbf{b} \rightarrow \mathbf{M} \cdot \mathbf{y} \leq \mathbf{d}]
$$

We extend the notion of partially bounded and unbounded universally quantified variables to QLIs. A Partially-bounded Quantified Linear Implication (PQLI) is a QLI in which every universally quantified variable is bounded only by a single absolute constraint. As with PQLPs, we can assume without loss of generality that this single constraint is a nonnegativity constraint. This constraint is placed in the LHS of the implication. Thus, a PQLI has the following form:

$$
\exists \mathbf{x}_{1} \forall \mathbf{y}_{1} \ldots \exists \mathbf{x}_{n} \forall \mathbf{y}_{n} \quad[(\mathbf{A} \cdot \mathbf{x} \leq \mathbf{b} \wedge \mathbf{y} \geq \mathbf{0}) \rightarrow \mathbf{C} \cdot \mathbf{x}+\mathbf{M} \cdot \mathbf{y} \leq \mathbf{d}]
$$

An Unbounded Quantified Linear Implication (UQLI) is a QLI in which no universally quantified variable appears in the LHS of the implication. Thus, a UQLI has the following form:

$$
\exists \mathbf{x}_{1} \forall \mathbf{y}_{1} \ldots \exists \mathbf{x}_{n} \forall \mathbf{y}_{n} \quad[\mathbf{A} \cdot \mathbf{x} \leq \mathbf{b} \rightarrow \mathbf{C} \cdot \mathbf{x}+\mathbf{M} \cdot \mathbf{y} \leq \mathbf{d}]
$$

It is important to note that 2-person game semantics have been proposed both for QLP [33] and QLI [14]. These are described in detail in Appendix B.1 and Appendix B.2 respectively.

A boolean formula $\phi$ is said to be in disjunctive normal form (DNF), if it consists of a disjunction of conjuncts, where each conjunct is a conjunction of literals. The formula is said to be in $3 \mathrm{DNF}$, if each conjunct has exactly three literals. 
A Quantified 3DNF (Q3DNF) formula is a quantified boolean expression of the form $Q(\mathbf{x}, \mathbf{y}) \phi(\mathbf{x}, \mathbf{y})$, where $Q(\mathbf{x}, \mathbf{y})$ is a quantifier string and $\phi(\mathbf{x}, \mathbf{y})$ is a boolean formula in 3DNF.

The following quantified boolean expression is an instance of Q3DNF:

$$
\exists x_{1} \forall y_{1} \exists x_{2} \forall y_{2} \exists x_{3} \forall y_{3} \quad\left(x_{1} \wedge y_{3} \wedge \neg x_{2}\right) \vee\left(x_{3} \wedge y_{2} \wedge \neg x_{1}\right) \vee\left(x_{2} \wedge y_{1} \wedge \neg x_{3}\right)
$$

We will use Q3DNFs to establish the connection between Quantified Linear Implication and the Polynomial Hierarchy. A 2-person game semantics for this problem is provided in Appendix B.3.

If $\mathbf{L}$ is a QLP, QLI or Q3DNF, the following terms are equivalent and used interchangeably:

1. L holds.

2. $\mathbf{L}$ is feasible.

3. $\mathbf{L}$ is satisfiable.

4. The existential player has a winning strategy for $\mathbf{L}$ (see Appendix B).

\section{Motivation}

Quantified Linear Programming represents a rich language that is ideal for expressing schedulability specifications in real-time scheduling $[8,16]$. In a real-time scheduling instance, a dispatcher typically determines whether a set of ordered, non-preemptive jobs can be scheduled within given time frames. Associated with each job is a start time and an execution time. The execution time of a job is a range-bound variable. There exist timing constraints that constrain the execution of jobs. These requirements can be modeled using QLPs.

For example, consider a real-time scheduling application with two jobs $J_{1}$ and $J_{2}$ with start times $s_{1}$ and $s_{2}$, and execution times $e_{1} \in\left[l_{e_{1}}, u_{e_{1}}\right]$ and $e_{2} \in\left[l_{e_{2}}, u_{e_{2}}\right]$. Assume that $J_{1}$ must start and finish before $J_{2}$ starts (i.e., $s_{1}+e_{1} \leq s_{2}$ ), while $J_{2}$ must start at most two time units after $J_{1}$ finishes (i.e., $s_{2} \leq s_{1}+e_{1}+2$ ). The corresponding QLP is the following:

$$
\begin{array}{r}
\exists s_{1} \forall e_{1} \in\left[l_{e_{1}}, u_{e_{1}}\right] \exists s_{2} \forall e_{2} \in\left[l_{e_{2}}, u_{e_{2}}\right] \\
s_{1}+e_{1}-s_{2} \leq 0, \\
s_{2}-\left(s_{1}+e_{1}\right) \leq 2
\end{array}
$$

Now, consider the case where the dispatcher has already obtained a schedule (solution). If new constraints are added to the specification, then the dispatcher may have to recompute the schedule. Alternatively, if it can be concluded that the current schedule does not cause violation of the newly added constraints, then the dispatcher can use the existing schedule. QLIs can be utilized to model the above decision problem. Note that QLP subsumes the two-level programming model discussed in [3].

QLPs and QLIs can be used to model reactive systems. A system is called reactive, if it maintains an ongoing interaction with its environment. A reactive system changes its actions, outputs, and status in response to the input it receives from the environment. Reactive systems are used in several real-world important applications and in various fields (see e.g., [17-19, 21, 23, 24]). QLP and QLI are important modeling tools for the design and implementation of reactive systems. The universally quantified variables can be used to represent the environmental input, while the existentially quantified variables can be used to represent the system's response. 


\section{Related work}

In this section, we briefly survey related work in the literature.

A decision procedure for the full elementary theory of real closed fields (see Appendix A.2) with addition $(+)$, multiplication $(\cdot)$ and order $(<,=)$ was established in [34]. Several quantifier elimination methods $[13,35]$ and efficient-in-practice approaches have been proposed since then $[5,9,11,12,25,26]$. The complexity of these quantifier elimination procedures is, in the worst case, doubly exponential in the number of quantifier alternations and exponential in the number of variables [10, 35].

Real numbers cannot be fully axiomatized by a first-order theory. Tarski's axiomatization of the reals requires a non-first-order axiom to express the Dedekind completeness of the real numbers (i.e., the property that asks all bounded subsets of real numbers to have a real least upper bound and a real greatest lower bound). The axiom in question involves universal quantification over subsets of the real numbers, which cannot be expressed in first-order logic.

Any field that satisfies all the same first-order properties as the real numbers is called a real closed field. Note that although the real algebraic numbers comprise a real closed field, they are not Dedekind complete. It is possible to construct a set of algebraic rational numbers, that has $\pi$, which is transcendental, as a supremum.

Several sub-classes of the full elementary theory of the reals have been studied.

The existential theory of the reals is obtained by restricting allowable expressions to existentially quantified formulas $\exists \mathbf{x} F(\mathbf{x})$ where $F(\mathbf{x})$ is a quantifier-free formula. There exists a decision procedure for this problem that is singly exponential in the number of quantified variables [1]. From the complexity perspective, it is known that this problem is NP-hard [30] and in PSPACE [7].

The theory of reals with addition and order (TLA) is obtained by restricting the set of function symbols to $\{+\}$. A quantifier elimination procedure for sentences in this theory that is singly exponential in space and doubly exponential in time is presented in [15]. An exponential time lower bound is shown in [2], where the time and space complexities at various levels of quantifier alternations are also determined.

Consider a formula in the theory of reals with addition and order in prenex normal form with $(k-1)$ quantifier alternations

$$
\exists \mathbf{x}_{1} \forall \mathbf{x}_{2} \ldots Q \mathbf{x}_{k} F\left(\mathbf{x}_{1}, \ldots, \mathbf{x}_{k}\right)
$$

where $Q$ is $\exists$ for $k$ odd and $\forall$ for $k$ even, while $F\left(\mathbf{x}_{1}, \ldots, \mathbf{x}_{k}\right)$ is a quantifier-free formula. This class of formulas has been proven to be log-space complete for $\Sigma_{\mathbf{k}} \mathbf{P}$ [31].

In this paper, we examine two interesting fragments of TLA:

(i) The Quantified Linear Programming fragment, where $F\left(\mathbf{x}_{1}, \ldots, \mathbf{x}_{k}\right)$ consists of conjunctions of linear inequalities, namely a linear system.

(ii) The Quantified Linear Implication fragment, where $F\left(\mathbf{x}_{1}, \ldots, \mathbf{x}_{k}\right)$ consists of a linear system implication, i.e., an implication between two linear systems.

QLP decidability is in PSPACE, though hardness has not yet been established [33]. The sub-classes of QLP with one quantifier alternation have the following complexities:

(i) If the first quantifier is existential, then the problem is in $\mathbf{P}$ [33].

(ii) If the first quantifier is universal, then the problem is coNP-complete [33].

Decidability of QLI with an arbitrary number of quantifier alternations has been shown to be PSPACE-hard [14]. The computational complexities of several sub-classes of QLI decidability with a given number of quantifier alternations have also been established [14]. 
Table 1 The computational complexity of deciding $\mathbb{R}, \mathbb{Q}, \mathrm{QLI}$, and QLP

\begin{tabular}{ll}
\hline Theory & Complexity \\
\hline $\mathbb{R}$ & EXPTIME-hard and 2-EXPTIME [10] \\
$\mathbb{R}^{(+, \leq)}=\mathbb{Q}=$ TLA & EXPTIME-hard [4] and 2-EXPTIME [15, 31] \\
QLI & PSPACE-hard [14] \\
QLP & coNP-hard and PSPACE [33] \\
\hline
\end{tabular}

Table 1 captures the various theories described above and the complexity of deciding the same. The corresponding inclusion diagram is provided in Fig. 1.

The principal contributions of this paper are presented below. We establish that:

(i) PQLP and UQLP decidability are in $\mathbf{P}$.

(ii) PQLI and UQLI decidability are in $\mathbf{P}$.

(iii) For each complexity class comprising the PH, there exists a class of QLI (i.e., an instantiation of the $\langle A, Q, T\rangle$ triple) which is complete for that class.

(iv) $\langle 1, \forall, \mathbf{R B}\rangle$ is in $\mathbf{P}$.

(v) $\langle 1, \exists, \mathbf{B L}\rangle$ is in $\mathbf{P}$.

(vi) $\langle 1, \forall, \mathbf{B R}\rangle$ and $\langle 1, \forall, \mathbf{B B}\rangle$ are coNP-complete.

\section{Variants of QLP}

In this section, we examine the computational complexities of PQLP and UQLP. We commence our analysis by showing that PQLP decidability is in $\mathbf{P}$.

\section{Theorem $1 P Q L P$ decidability is in $\mathbf{P}$.}

Proof Let $\mathbf{L}$ be the following PQLP:

$$
\begin{array}{r}
\exists x_{n} \forall y_{n} \in[0,+\infty) \exists x_{n-1} \forall y_{n-1} \in[0,+\infty) \ldots \exists x_{1} \\
\forall y_{1} \in[0,+\infty) \exists x_{0} \mathbf{A} \cdot \mathbf{x}+\mathbf{B} \cdot \mathbf{y} \leq \mathbf{c}
\end{array}
$$

Let $\mathbf{A}=\left(\mathbf{a}_{n}, \mathbf{a}_{n-1}, \ldots, \mathbf{a}_{0}\right), \mathbf{B}=\left(\mathbf{b}_{n}, \mathbf{b}_{n-1}, \ldots, \mathbf{b}_{1}\right), \mathbf{x}=\left(x_{n}, x_{n-1}, \ldots, x_{0}\right)^{T}$, and $\mathbf{y}=\left(y_{n}, y_{n-1}, \ldots, y_{1}\right)^{T}$. Note that any PQLP can be reduced to the form specified by PQLP (9) through the addition of dummy variables. Enforcing this strict alternation of quantifiers will at most double the total number of program variables.

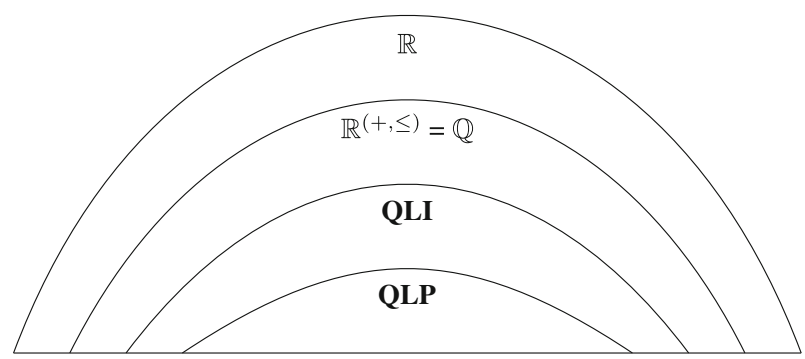

Fig. 1 Inclusion diagram of $\mathbb{R}, \mathbb{Q}, \mathrm{QLI}$, and QLP 
Consider the linear program:

$$
L P_{n}: \mathbf{A} \cdot \mathbf{x} \leq \mathbf{c},
$$

and, for $i=1, \ldots, n$ consider the linear program:

$$
\begin{array}{r}
L P_{i-1}: \mathbf{A} \cdot \mathbf{x}+\mathbf{b}_{i} \leq \mathbf{0} \\
x_{i}, \ldots, x_{n}=0 .
\end{array}
$$

We will show that PQLP (9) is feasible if and only if $L P_{n}$ is feasible, and $L P_{i-1}$ is feasible for all $i=1 \ldots n$.

Assume that the linear program represented by System (10) and the linear programs represented by System (11) are feasible.

Let $\hat{\mathbf{x}}^{n}$ denote a solution to $L P_{n}$. Furthermore, let $\hat{\mathbf{x}}^{i-1}$ denote a solution to $L P_{i-1}$ for $i=1, \ldots, n$.

Let $\mathbf{x}^{\mathbf{s}}$ denote the strategy of the existential player $\mathbf{X}$ and let $\mathbf{y}^{\mathrm{s}}$ denote the strategy of the universal player $\mathbf{Y}$.

Consider the assignment

$$
\mathbf{x}^{\mathbf{s}}=\hat{\mathbf{x}}^{n}+\sum_{i=1}^{n} y_{i} \cdot \hat{\mathbf{x}}^{i-1}
$$

Note that in $L P_{i-1}$, the constraints $x_{i}, \ldots, x_{n}=0$ ensure that $x_{n}^{s}$ through $x_{i}^{s}$ do not depend on the values of $y_{i}$ through $y_{1}$. Hence, the moves specified by the strategy $\mathbf{x}^{\mathbf{s}}$ depend only on the moves previously made by $\mathbf{Y}$, i.e., System (12) is a strategy for $\mathbf{X}$.

As per the 2-person game semantics of QLPs, the game corresponding to System (9) will be played as follows:

1. $\mathbf{X}$ chooses $x_{n}=x_{n}^{s}=\hat{x}_{n}^{n}$.

2. $\mathbf{Y}$ chooses $y_{n}=y_{n}^{s} \in[0, \infty) ; \mathbf{X}$ chooses $x_{n-1}=x_{n-1}^{s}=\hat{x}_{n-1}^{n}+y_{n} \cdot \hat{x}_{n-1}^{n-1}$.

3. $\mathbf{Y}$ chooses $y_{i+1}=y_{i+1}^{s} \in[0, \infty) ; \mathbf{X}$ chooses $x_{i}=x_{i}^{s}=\hat{x}_{i}^{n}+\sum_{j=i}^{n-1} y_{j+1} \cdot \hat{x}_{i}^{j}$.

4. $\mathbf{Y}$ chooses $y_{1}=y_{1}^{s} \in[0, \infty) ; \mathbf{X}$ chooses $x_{0}=x_{0}^{s}=\hat{x}_{0}^{n}+\sum_{j=0}^{n-1} y_{j+1} \cdot \hat{x}_{0}^{j}$.

Observe that all the $x_{i}^{s}, i=0, \ldots, n$ and the $y_{i}^{s}, i=1, \ldots, n$ are assigned linear values. Let $\mathbf{x}_{\mathbf{T}}$ be the numeric vector assigned to $\mathbf{x}$ by the strategy $\mathbf{x}^{\mathbf{s}}$. It follows that $\mathbf{x}_{\mathbf{T}}=\hat{\mathbf{x}}^{n}+$ $\sum_{i=1}^{n} y_{i}^{\mathbf{s}} \cdot \hat{\mathbf{x}}^{i-1}$. Likewise, let $\mathbf{y}_{\mathbf{T}}=\left(y_{n}^{\mathbf{s}}, y_{n-1}^{\mathbf{s}}, \ldots, y_{1}^{\mathbf{s}}\right)$. Accordingly, the outcome of the play can be evaluated as:

$$
\begin{aligned}
\mathbf{A} \cdot \mathbf{x}_{\mathbf{T}}+\mathbf{B} \cdot \mathbf{y}_{\mathbf{T}} & =\mathbf{A} \cdot\left(\hat{\mathbf{x}}^{n}+\sum_{i=1}^{n} y_{i}^{\mathbf{s}} \cdot \hat{\mathbf{x}}^{i-1}\right)+\sum_{i=1}^{n} \mathbf{b}_{i} \cdot y_{i}^{\mathbf{s}} \\
& =\mathbf{A} \cdot \hat{\mathbf{x}}^{n}+\sum_{i=1}^{n} y_{i}^{\mathbf{s}} \cdot\left(\mathbf{A} \cdot \hat{\mathbf{x}}^{i-1}+\mathbf{b}_{i}\right) \\
& \leq \mathbf{c}+\sum_{i=1}^{n} y_{i}^{\mathbf{s}} \cdot \mathbf{0} \\
& =\mathbf{c}
\end{aligned}
$$

Since Y's strategy was chosen arbitrarily, at the end of any play, X's strategy forces $\mathbf{A} \cdot \mathbf{x}+$ B $\cdot \mathbf{y} \leq \mathbf{c}$. Thus, PQLP (9) is feasible, proving our claim. 
Now assume that PQLP (9) is feasible. Thus, $\mathbf{X}$ has a winning strategy $\mathbf{x}^{\mathbf{s}}$.

Consider the case when $\mathbf{Y}$ plays according to the strategy $\mathbf{y}^{\mathbf{s}}=\mathbf{0}$. Let $\mathbf{x}^{*}$ be the numeric vector assigned to $\mathbf{x}$ by the strategy $\mathbf{x}^{\mathbf{s}}$ in this situation. It is clear that $\mathbf{x}^{*}$ satisfies $L P_{n}$.

Let us focus on a specific value of $i$. Let $\overline{\mathbf{y}}^{i}=(0,0, \ldots, 1, \ldots, 0)^{T}$. Note that $\bar{y}_{i}^{i}=1$ is the only non-zero element of $\overline{\mathbf{y}}^{i}$.

Assume that $L P_{i-1}$ is infeasible. Let $\mathbf{x}^{i}=\left(x_{i-1}, \ldots, x_{0}\right)$ and let $\mathbf{A}^{i}$ be the matrix consisting of the corresponding columns of $\mathbf{A}$. Note that we can rewrite $L P_{i-1}$ as $\mathbf{A}^{i} \cdot \mathbf{x}^{i} \leq-\mathbf{b}_{i}$. By Farkas' Lemma, there exists a vector $\mathbf{z} \in \mathbb{R}_{+}^{m}$ such that $\mathbf{z}^{T} \cdot \mathbf{A}^{i}=0$ and $-\mathbf{z}^{T} \cdot \mathbf{b}_{i}<0$. We can assume without loss of generality that $\mathbf{z}^{T} \cdot \mathbf{b}_{i}=1$.

Let $M \in \mathbb{Z}^{+}$denote an arbitrary integer constant. Consider the case when $\mathbf{Y}$ plays according to the strategy $M \cdot \overline{\mathbf{y}}^{i}$. Let $\mathbf{x}^{\prime}$ be the numeric vector assigned to $\mathbf{x}$ by the strategy $\mathbf{x}^{\mathbf{s}}$ in this situation. Let $\overline{\mathbf{x}}^{i M}$ be the numeric vector such that $\mathbf{x}^{\prime}=\left(\mathbf{x}^{*}+\overline{\mathbf{x}}^{i M}\right)$. Thus $\overline{\mathbf{x}}^{i M}$ represents the change in $\mathbf{X}$ 's response when $\mathbf{Y}^{\prime}$ 's play changes by $M \cdot \overline{\mathbf{y}}^{i}$.

Until $y_{i}$ is assigned a value, Y's plays (viz., $\mathbf{0}$ and $M \cdot \overline{\mathbf{y}}^{i}$ ) are indistinguishable. Thus, $\mathbf{X}$ 's response cannot change until after $y_{i}$ is assigned a value. This means that $\bar{x}_{i}^{i M}, \ldots, \bar{x}_{n}^{i M}=0$.

We have $\mathbf{A} \cdot \mathbf{x}^{*} \leq \mathbf{c}$ and $\mathbf{A} \cdot\left(\mathbf{x}^{*}+\overline{\mathbf{x}}^{i M}\right)+\mathbf{B} \cdot\left(M \cdot \overline{\mathbf{y}}^{i}\right) \leq \mathbf{c}$. Since $M$ was chosen arbitrarily, we have:

$$
(\forall M)\left(\mathbf{A} \cdot \mathbf{x}^{*}\right)+\mathbf{A} \cdot \overline{\mathbf{x}}^{i M}+M \cdot\left(\mathbf{B} \cdot \overline{\mathbf{y}}^{i}\right) \leq \mathbf{c} .
$$

Since $\bar{x}_{i}^{i M}, \ldots, \bar{x}_{n}^{i M}=0$, we can rewrite $\mathbf{A} \cdot \overline{\mathbf{x}}^{i M}$ as $\mathbf{A}^{i} \cdot \overline{\mathbf{x}}^{i M}$. Thus System (13) can be rewritten as:

$$
(\forall M)\left(\mathbf{A} \cdot \mathbf{x}^{*}\right)+\mathbf{A}^{i} \cdot \overline{\mathbf{x}}^{i M}+M \cdot \mathbf{b}_{i} \leq \mathbf{c} .
$$

We can now multiply System (14) by $\mathbf{z}^{T}$. Since $\mathbf{z}$ is non-negative we get:

$$
\begin{aligned}
(\forall M) \mathbf{z}^{T} \cdot\left(\mathbf{A} \cdot \mathbf{x}^{*}\right)+\mathbf{z}^{T} \cdot\left(\mathbf{A}^{i} \cdot \overline{\mathbf{x}}^{i M}+M \cdot \mathbf{b}_{i}\right) & \leq \mathbf{z}^{T} \cdot \mathbf{c} \\
(\forall M) \mathbf{z}^{T} \cdot\left(\mathbf{A} \cdot \mathbf{x}^{*}\right)+\left(\left(\mathbf{z}^{T} \cdot \mathbf{A}^{i}\right) \cdot \overline{\mathbf{x}}^{i M}+M \cdot \mathbf{z}^{T} \cdot \mathbf{b}_{i}\right) & \leq \mathbf{z}^{T} \cdot \mathbf{c} \\
(\forall M) \mathbf{z}^{T} \cdot\left(\mathbf{A} \cdot \mathbf{x}^{*}\right)+\left(\mathbf{0} \cdot \overline{\mathbf{x}}^{i M}+M\right) & \leq \mathbf{z}^{T} \cdot \mathbf{c} \\
(\forall M) M & \leq \mathbf{z}^{T} \cdot\left(\mathbf{c}-\mathbf{A} \cdot \mathbf{x}^{*}\right) .
\end{aligned}
$$

This is clearly infeasible. Thus, $L P_{i-1}$ must be feasible. Since $i$ was chosen arbitrarily, we have that this is true for every $i=1 \ldots n$.

Thus, we have successfully reduced the problem of deciding the feasibility of PQLP (9) to the problem of deciding the feasibility of $L P_{n}$ and the feasibility of $L P_{i-1}$ for $i=1 \ldots n$. Since the problem of deciding LP feasibility is in $\mathbf{P}$ [20], it follows that the problem of deciding PQLP feasibility is also in $\mathbf{P}$.

We now show that UQLP decidability is in $\mathbf{P}$.

\section{Theorem 2 UQLP decidability is in $\mathbf{P}$.}

Proof Consider the following UQLP:

$$
\exists \mathbf{x}_{\mathrm{n}} \forall \mathbf{y}_{\mathrm{n}} \exists \mathbf{x}_{\mathrm{n}-1} \forall \mathbf{y}_{\mathrm{n}-1} \ldots \exists \mathbf{x}_{1} \forall \mathbf{y}_{1} \exists \mathbf{x}_{0} \quad \mathbf{A} \cdot \mathbf{x}+\mathbf{B} \cdot \mathbf{y} \leq \mathbf{c}
$$

To show that UQLP (15) can be solved in polynomial time, we reduce it to a PQLP. 
Consider the following PQLP:

$$
\begin{gathered}
\exists \mathbf{x}_{\mathrm{n}} \forall \mathbf{y}_{\mathrm{n}}^{\prime} \in[0,+\infty) \forall \mathbf{y}_{\mathrm{n}}^{\prime \prime} \in[0,+\infty) \exists \mathbf{y}_{\mathrm{n}} \exists \mathbf{x}_{n-1} \\
\forall \mathbf{y}_{\mathrm{n}-1}^{\prime} \in[0,+\infty) \forall \mathbf{y}_{\mathrm{n}-1}^{\prime \prime} \in[0,+\infty) \exists \mathbf{y}_{\mathrm{n}-1} \ldots \\
\exists \mathbf{x}_{1} \forall \mathbf{y}_{1}^{\prime} \in[0,+\infty) \forall \mathbf{y}_{1}^{\prime \prime} \in[0,+\infty) \exists \mathbf{y}_{1} \exists \mathbf{x}_{0} \\
\mathbf{A} \cdot \mathbf{x}+\mathbf{B} \cdot \mathbf{y} \leq \mathbf{c} \\
\mathbf{y}_{\mathrm{i}}=\mathbf{y}_{\mathrm{i}}^{\prime}-\mathbf{y}_{\mathrm{i}}^{\prime \prime}, i=1,2, \ldots n .
\end{gathered}
$$

In PQLP (16), $\mathbf{y}_{1}^{\prime}, \ldots, \mathbf{y}_{\mathrm{n}}^{\prime}$ is a partition of $\mathbf{y}^{\prime}$ and $\mathbf{y}_{1}^{\prime \prime}, \ldots, \mathbf{y}_{n}^{\prime \prime}$ is a partition of $\mathbf{y}^{\prime \prime}$, such that $\left|\mathbf{y}_{i}^{\prime}\right|=\left|\mathbf{y}_{i}^{\prime \prime}\right|=\left|\mathbf{y}_{i}\right|$, for $i=1 \ldots n$.

The key observation is that the universal player can win System (15) if and only if he can win System (16). To see this, note that the value of $\mathbf{y}_{\mathbf{i}}$ in System (16) is completely determined by the universal player's choices for $\mathbf{y}_{\mathrm{i}}^{\prime}$ and $\mathbf{y}_{\mathrm{i}}^{\prime \prime}$. Since $\mathbf{y}_{i}^{\prime} \in[\mathbf{0},+\infty)$ and $\mathbf{y}_{i}^{\prime \prime} \in$ $[\mathbf{0},+\infty)$, $\mathbf{y}$ can take any value in the interval $(-\infty,+\infty)$. Thus, the quantifier sequence $\forall \mathbf{y}_{\mathrm{i}}$ in System (15) $i=1,2, \ldots, n$, can be replaced respectively by the quantifier sequence $\forall \mathbf{y}_{\mathbf{i}}^{\prime} \in[\mathbf{0},+\infty) \forall \mathbf{y}_{\mathbf{i}}^{\prime \prime} \in[\mathbf{0},+\infty) \exists \mathbf{y}_{\mathrm{i}}$ to get System (16), without affecting its feasibility. In other words, UQLP (15) is feasible if and only is PQLP (16) is feasible. From Theorem 1, it follows that UQLP decidability is in $\mathbf{P}$.

By proving that UQLP is in $\mathbf{P}$, we have essentially shown the following: The computational complexity of deciding a formula in TLA depends on both the number of alternations in the quantifier specification and the syntactic restriction. Observe that in the absence of any syntactic restriction, and in the presence of unbounded alternation, the TLA decidability problem is in 2-EXPTIME [15, 31] and EXPTIME-hard [4]. However, the conjunctive fragment of TLA, even with unbounded alternation, is decidable in polynomial time.

\section{Variants of QLI}

We utilize the results of Section 5 to show that PQLI and UQLI are also solvable in polynomial time.

\section{Corollary 1 PQLI decidability is in $\mathbf{P}$.}

Proof Consider the following PQLI:

$$
\exists \mathbf{x}_{1} \forall \mathbf{y}_{1} \ldots \exists \mathbf{x}_{\mathrm{n}} \forall \mathbf{y}_{\mathrm{n}}[\mathbf{A} \cdot \mathbf{x} \leq \mathbf{b}, \mathbf{y} \geq \mathbf{0} \rightarrow \mathbf{C} \cdot \mathbf{x}+\mathbf{D} \cdot \mathbf{y} \leq \mathbf{f}]
$$

We will focus on the following two cases:

1. There exists a point $\mathbf{z}$, such that $\mathbf{A} \cdot \mathbf{z} \not \leq \mathbf{b}$ - It follows that $\forall \mathbf{x}(\mathbf{A} \cdot \mathbf{x} \leq \mathbf{b})$ does not hold. In this case, the PQLI is trivially satisfied, since the existential player can guess $\mathbf{z}$ and cause the left hand side of the implication to be falsified.

2. There is no point $\mathbf{z}$ such that $\mathbf{A} \cdot \mathbf{z} \not \leq \mathbf{b}$ - In this case, the PQLI (17) reduces to the following PQLI.

$$
\exists \mathbf{x}_{1} \forall \mathbf{y}_{1} \ldots \exists \mathbf{x}_{\mathrm{n}} \forall \mathbf{y}_{\mathrm{n}} \quad[\mathbf{y} \geq \mathbf{0} \rightarrow \mathbf{C} \cdot \mathbf{x}+\mathbf{D} \cdot \mathbf{y} \leq \mathbf{f}]
$$

PQLI (18) can in turn be written as:

$$
\exists \mathbf{x}_{1} \forall \mathbf{y}_{1} \in[\mathbf{0},+\infty) \ldots \exists \mathbf{x}_{\mathrm{n}} \forall \mathbf{y}_{\mathrm{n}} \in[\mathbf{0},+\infty) \mathbf{C} \cdot \mathbf{x}+\mathbf{D} \cdot \mathbf{y} \leq \mathbf{f}
$$


However, System (19) is a PQLP, and hence can be decided in polynomial time by Theorem 1.

\section{Corollary 2 UQLI decidability is in $\mathbf{P}$.}

Proof Consider the following UQLI:

$$
\exists \mathbf{x}_{1} \forall \mathbf{y}_{1} \ldots \exists \mathbf{x}_{\mathrm{n}} \forall \mathbf{y}_{\mathrm{n}}[\mathbf{A} \cdot \mathbf{x} \leq \mathbf{b} \rightarrow \mathbf{C} \cdot \mathbf{x}+\mathbf{D} \cdot \mathbf{y} \leq \mathbf{f}]
$$

As argued in the proof of Corollary 1, if $\forall \mathbf{x}(\mathbf{A} \cdot \mathbf{x} \leq \mathbf{b})$ does not hold, then UQLI (20) is trivially satisfied.

Otherwise, UQLI (20) is equivalent to:

$$
\exists \mathbf{x}_{1} \forall \mathbf{y}_{1} \ldots \exists \mathbf{x}_{\mathrm{n}} \forall \mathbf{y}_{\mathrm{n}} \mathbf{C} \cdot \mathbf{x}+\mathbf{D} \cdot \mathbf{y} \leq \mathbf{f}
$$

which is a UQLP, and hence can be decided in polynomial time by Theorem 2 .

\section{QLI and the polynomial hierarchy}

In this section, we prove that for each class of the PH, there exists a class of QLI decidability that is complete for that class. This is interesting, since QLIs are comprised of continuous variables, as opposed to the discrete variables comprising QBFs. Hence, we provide a continuous analogue to the results in [32], where the $\mathbf{P H}$ is generated using QBFs.

Let $\mathbf{B}^{k+1}$ denote a string of $(k+1)$ Bs. The following results were obtained in [14].

Theorem $3\left\langle k, \exists, \mathbf{B}^{k+1}\right\rangle$ with $k$ odd, is $\Sigma_{\mathbf{k}} \mathbf{P}$-hard.

Theorem $4\left\langle k, \forall, \mathbf{B}^{k+1}\right\rangle$ with $k$ even, is $\Pi_{\mathbf{k}} \mathbf{P}$-hard.

To establish the computational complexities of $\left\langle k, \exists, \mathbf{B}^{k+1}\right\rangle$ when $k$ is even, and $\left\langle k, \forall, \mathbf{B}^{k+1}\right\rangle$ when $k$ is odd, we first provide a reduction from Q3DNF to QLI.

\subsection{Reduction from Q3DNF to QLI}

Consider a Q3DNF instance $\boldsymbol{\Phi}: Q(\mathbf{x}, \mathbf{y}) \phi(\mathbf{x}, \mathbf{y})$, where $Q(\mathbf{x}, \mathbf{y})$ represents the quantifier string, $\mathbf{x}$ is the set of existentially quantified variables, and $\mathbf{y}$ is the set of universally quantified variables. Note that $\phi(\mathbf{x}, \mathbf{y})=\phi_{1} \wedge \phi_{2} \wedge \ldots \wedge \phi_{m}$ where each $\phi_{i}$ is a disjunctive clause. Without loss of generality, we can assume that the innermost quantifier of $Q(\mathbf{x}, \mathbf{y})$ is $\forall$, since if the innermost quantifier is $\exists$, then this variable can be eliminated in polynomial time. This is because Q3DNF is the complement of Q3CNF and an innermost quantifier of $\forall$ can be eliminated from a Q3CNF formula in polynomial time [22].

We will produce a QLI I : $Q^{\prime}(\mathbf{x}, \mathbf{r}, \mathbf{y}, \mathbf{s}, \mathbf{w}) E \rightarrow F$, such that the existential player has a winning strategy for $\mathbf{I}$, if and only if the existential player has a winning strategy for $\boldsymbol{\Phi}$. Note that $E$ represents the set of constraints on the LHS of the constructed implication, and $F$ represents the set of constraints on the RHS of the implication.

For each existentially quantified variable $x_{i}$ in $\boldsymbol{\Phi}$, we add an existentially quantified variable $x_{i}$ and a universally quantified variable $r_{i}$ to $\mathbf{I}$. We also add the constraints $r_{i} \leq x_{i}$ and $r_{i} \leq 1-x_{i}$ to $E$, and the constraint $r_{i} \leq 0$ to $F$. Note that these constraints are equivalent to $r_{i} \leq \min \left(x_{i}, 1-x_{i}\right) \rightarrow r_{i} \leq 0$. Finally, we add the constraint $0 \leq x_{i} \leq 1$ to $F$. 
For each universally quantified variable $y_{i}$ in $\boldsymbol{\Phi}$, we add an existentially quantified variable $s_{i}$ and a universally quantified variable $y_{i}$ to $\mathbf{I}$. We also add the constraints $0 \leq y_{i} \leq 1$ to $E$, and the constraints $0 \leq s_{i} \leq 1,2 \cdot y_{i}-1 \leq s_{i}$, and $s_{i} \leq 2 \cdot y_{i}$ to $F$. Note that these are the only constraints that use $y_{i}$ variables, since the clause constraints use only $x_{i}$ and $s_{i}$ variables.

For each clause $\phi_{j}$ in $\phi(\mathbf{x}, \mathbf{y})$, we add the existentially quantified variable $w_{j}$ to $\mathbf{I}$, and 3 constraints to $F$. These constraints ensure that $w_{j}$ is less than or equal to the existential variables corresponding to the literals in $\phi_{j}$. Note that these constraints contain only existential variables. Even in the case of a universal variable $y_{i}$ in $\phi_{j}$, the constraint contains the existential variable $s_{i}$ of the QLI.

For example, if $\phi_{j}=\left(x_{i}, y_{k}, \bar{x}_{l}\right)$, we add $w_{j} \leq x_{i}, w_{j} \leq s_{k}$, and $w_{j} \leq 1-x_{l}$ to $F$, while if $\phi_{j}=\left(x_{i}, \bar{y}_{k}, \bar{x}_{l}\right)$, we add $w_{j} \leq x_{i}, w_{j} \leq 1-s_{k}$, and $w_{j} \leq 1-x_{l}$ to $F$.

Finally, we add the linear constraint $w_{1}+w_{2}+\cdots+w_{m} \geq 1$ to $F$.

We inductively create the quantifier string $Q^{\prime}(\mathbf{x}, \mathbf{r}, \mathbf{y}, \mathbf{s}, \mathbf{w})$ of $\mathbf{I}$ based on $Q(\mathbf{x}, \mathbf{y})$ : $Q^{\prime}(\mathbf{x}, \mathbf{r}, \mathbf{y}, \mathbf{s}, \mathbf{w})$ of $\mathbf{I}$ is initialized to $\epsilon$ (the empty string). For each $i=1,2, \ldots, n$,

1. If the $i^{t h}$ quantifier of $Q(\mathbf{x}, \mathbf{y})$ is $\exists \mathbf{x}_{i}$, then we append $\exists \mathbf{x}_{i} \forall \mathbf{r}_{i}$ to the end of $Q^{\prime}(\mathbf{x}, \mathbf{r}, \mathbf{y}, \mathbf{s}, \mathbf{w})$.

2. If the $i^{t h}$ quantifier of $Q(\mathbf{x}, \mathbf{y})$ is $\forall \mathbf{y}_{i}$, then we append $\forall \mathbf{y}_{i} \exists \mathbf{s}_{i}$ to the end of $Q^{\prime}(\mathbf{x}, \mathbf{r}, \mathbf{y}, \mathbf{s}, \mathbf{w})$.

Finally, we add $\exists \mathbf{w}$ to the end of $Q^{\prime}(\mathbf{x}, \mathbf{r}, \mathbf{y}, \mathbf{s}, \mathbf{w})$.

Since the final quantifier of $Q(\mathbf{x}, \mathbf{y})$ is $\forall$, the number of quantifier alternations in

$Q^{\prime}(\mathbf{x}, \mathbf{r}, \mathbf{y}, \mathbf{s}, \mathbf{w})$ is one more than the number of quantifier alternations in $Q(\mathbf{x}, \mathbf{y})$.

Note that the final quantifier of $Q^{\prime}(\mathbf{x}, \mathbf{r}, \mathbf{y}, \mathbf{s}, \mathbf{w})$ is $\exists$.

Example 4 Let us consider the Q3DNF instance

$$
\boldsymbol{\Phi}=\exists x_{1} \forall y_{1} \exists x_{2} \forall y_{2} \underbrace{\left(x_{1}, \bar{y}_{1}, y_{2}\right)}_{\phi_{1}} \vee \underbrace{\left(\bar{x}_{1}, x_{2}, y_{2}\right)}_{\phi_{2}} .
$$

Under the above construction, $\boldsymbol{\Phi}$ becomes an instance of $\langle 4, \exists, \mathbf{B B B B R}\rangle$ with the quantifier string

$$
\exists x_{1} \forall r_{1} \forall y_{1} \exists s_{1} \exists x_{2} \forall r_{2} \forall y_{2} \exists s_{2} \exists w_{1} \exists w_{2} .
$$

Note that this is also an instance of $\langle 4, \exists, \mathbf{B B B B B}\rangle$ [14].

The LHS of the implication $(E)$ consists of the following set of constraints:

$$
\begin{aligned}
r_{1} \leq x_{1}, & r_{1} \leq 1-x_{1}, \\
r_{2} \leq x_{2}, & r_{2} \leq 1-x_{2}, \\
0 \leq y_{1} \leq 1, & 0 \leq y_{2} \leq 1,
\end{aligned}
$$

The RHS of the implication $(F)$ consists of the following set of constraints:

$$
\begin{aligned}
& r_{1} \leq 0, \quad 0 \leq x_{1} \leq 1, \\
& r_{2} \leq 0, \quad 0 \leq x_{2} \leq 1, \\
& 0 \leq s_{1} \leq 1, \quad 0 \leq s_{2} \leq 1, \\
& 2 \cdot y_{1}-1 \leq s_{1}, \quad s_{1} \leq 2 \cdot y_{1} \text {, } \\
& 2 \cdot y_{2}-1 \leq s_{2}, \quad s_{2} \leq 2 \cdot y_{2}, \\
& \phi_{1}\left\{\begin{aligned}
w_{1} \leq x_{1}, & w_{2} \leq 1-x_{1}, \\
w_{1} \leq 1-s_{1}, & w_{2} \leq x_{2}, \\
w_{1} \leq s_{2}, & w_{2} \leq s_{2},
\end{aligned}\right\} \phi_{2} \\
& w_{1}+w_{2} \geq 1 \text {. }
\end{aligned}
$$


We now establish that all the variables in $\mathbf{I}$ can be restricted to $\{0,1\}$ without affecting its feasibility. To do so, we utilize the game semantics discussed in Appendix B.

Lemma 1 The existential player $\mathbf{X}$ has a winning strategy, if and only if he has a winning strategy, when each $x_{i}$ is chosen from $\{0,1\}$.

Proof The if part is obvious.

Accordingly, we focus on proving the only if part. Assume that the existential player $\mathbf{X}$ has a winning strategy. First, observe that if $x_{i} \notin[0,1]$, then at least one of the constraints in $F$ is violated. In particular, the constraint $0 \leq x_{i} \leq 1$ is violated. Now focus on the constraints in $E$. Since each $r_{i}$ is chosen by the universal player $\mathbf{Y}$ after the existential player chooses $x_{i}$, these constraints can only be violated by $\mathbf{Y}$. In other words $\mathbf{Y}$ can always satisfy the RHS of the implication. Thus, the implication does not hold and $\mathbf{X}$ does not have a winning strategy.

We now consider the case $x_{i} \in(0,1)$. In this case, $\mathbf{Y}$ could choose $r_{i}=\min \left(x_{i}, 1-x_{i}\right)$ $>0$. Note that both $x_{i}$ and $r_{i}$ are positive with $r_{i} \leq x_{i}$ and $r_{i} \leq 1-x_{i}$. Thus the universal player $\mathbf{Y}$ wins the game, since the constraints involving $x_{i}$ and $r_{i}$ in $E$, viz., $r_{i} \leq x_{i}$ and $r_{i} \leq 1-x_{i}$ are satisfied and the constraint $r_{i} \leq 0$ in $F$ is violated.

To sum up, any choice of $x_{i} \notin\{0,1\}$ would cause $\mathbf{X}$ to lose the game.

It follows that if $\mathbf{X}$ has a winning strategy, then he has a winning strategy when each $x_{i}$ is chosen from $\{0,1\}$.

Lemma 2 The universal player $\boldsymbol{Y}$ has a winning strategy, if and only if he has a winning strategy, when each $y_{i}$ is chosen from $\{0,1\}$.

Proof The if part is obvious.

Accordingly, we focus on proving the only-if part. It is clear that in order to win, the universal player $\mathbf{Y}$ must choose $y_{i} \in[0,1]$. Otherwise, the constraint $0 \leq y_{i} \leq 1$ in the LHS $E$ of the implication is violated and the existential player $\mathbf{X}$ wins the game.

Suppose now that $\mathbf{Y}$ can win by choosing $y_{i} \in(0,1)$. As per the construction of Section 7.1, the only constraints involving $s_{i}$ and $y_{i}$ are given by System (21).

$$
0 \leq s_{i} \leq 1,2 \cdot y_{i}-1 \leq s_{i}, s_{i} \leq 2 \cdot y_{i}
$$

If $y_{i} \in\left(0, \frac{1}{2}\right]$, then from System (21) it follows that $s_{i}$ can take any value in $\left[0,2 \cdot y_{i}\right]$. However, if instead $\mathbf{Y}$ had chosen $y_{i}=0$, then $s_{i}$ is forced to equal 0 . It follows that if $\mathbf{Y}$ can win by choosing $y_{i} \in\left(0, \frac{1}{2}\right]$, then $\mathbf{Y}$ can win by choosing $y_{i}=0$. Similarly, if $\mathbf{Y}$ can win by choosing $y_{i} \in\left[\frac{1}{2}, 1\right)$, then $\mathbf{Y}$ can win by choosing $y_{i}=1$.

To sum up, we can safely assume that the universal player only chooses $y_{i} \in\{0,1\}$.

It follows that if $\mathbf{Y}$ has a winning strategy, then he has a winning strategy when each $y_{i}$ is chosen from $\{0,1\}$.

The import of Lemma 1 and Lemma 2 is that we can confine our analyses to games in which the existential player $\mathbf{X}$ and universal player $\mathbf{Y}$ make moves in $\{0,1\}$ for the $x_{i}$ and $y_{i}$ variables respectively.

An interesting observation is that if $y_{i}$ is restricted to $\{0,1\}$, then so is $s_{i}$. The constraints involving $s_{i}$ and $y_{i}$ in the RHS $F$ of the implication are described by System (21). If $y_{i}=0$, the constraints in System (21) force $s_{i}$ to be 0 ; likewise, if $y_{i}=1$, they force $s_{i}$ to be 1 . In other words, $\mathbf{X}$ is forced to set $s_{i}=y_{i}$. Any other choice of $s_{i}$ would violate at least one 
constraint of $F$, causing $\mathbf{X}$ to lose the game. Hence, the $s_{i}$ variables are also restricted in the set $\{0,1\}$.

Theorem $5 \mathbf{I}$ is feasible if and only if $\boldsymbol{\Phi}$ is feasible.

Proof We show that the existential player $\mathbf{X}$ has a winning strategy for $\mathbf{I}$ if and only if he has a winning strategy for $\boldsymbol{\Phi}$.

Only if part: Assume that the existential player $\mathbf{X}$ has a winning strategy for $\mathbf{I}$. Consider a play of the game, in which $\mathbf{X}$ chooses values for the existentially quantified variables and $\mathbf{Y}$ chooses values for the universally quantified variables. After $\mathbf{X}$ chooses a value for $x_{i}$, $\mathbf{Y}$ can choose $r_{i}$, such that the constraints $r_{i} \leq x_{i}$ and $r_{i} \leq 1-x_{i}$ are both satisfied. By our 2-person game semantics, the universal player $\mathbf{Y}$ will ensure that all the constraints in the LHS are satisfied. In order for $\mathbf{X}$ to win the game, he has to ensure that all the constraints in the RHS are satisfied as well. In particular, the constraint $w_{1}+w_{2}+\cdots+w_{m} \geq 1$ in the RHS of the implication must be satisfied. Consequently, $w_{j}>0$, for at least one $j$. This $w_{j}$ corresponds to the clause $\phi_{j}$ of $\boldsymbol{\Phi}$. Assume that $\phi_{j}$ has the form $\left(x_{i}, y_{k}, x_{l}\right)$. Since the $x_{i}$ s and $s_{i}$ s are restricted to the set $\{0,1\}$ (see Lemma 1 and Lemma 2), the constraints $w_{j} \leq x_{i}, w_{j} \leq s_{k}$, and $w_{j} \leq x_{l}$ force each variable $\left(x_{i}, s_{k}\right.$ and $\left.x_{l}\right)$ to be 1 . It follows that $\phi_{j}$ is satisfied in this play. Similar arguments can be made for other forms of $\phi_{j}$. Since the play was chosen arbitrarily, the same argument applies for all plays, i.e., at least one clause of $\phi(\mathbf{x}, \mathbf{y})$ is satisfied in every play. Hence, $\boldsymbol{\Phi}$ is feasible.

If part: Assume that the existential player $\mathbf{X}$ has a winning strategy for $\boldsymbol{\Phi}$.

At the end of any play, $\phi(\mathbf{x}, \mathbf{y})$ is satisfied. Thus, at least one clause, say $\phi_{j}$, must be satisfied. Assume that $\phi_{j}$ is of the form $\left(x_{i}, y_{k}, x_{l}\right)$. Consider the constraints constructed from $\phi_{j}$, viz., $w_{j} \leq x_{i}, w_{j} \leq s_{k}$, and $w_{j} \leq x_{l}$. Since $\phi_{j}$ is satisfied, $x_{i}, y_{k}, x_{l}$ are all true. Assume that $\mathbf{X}$ sets the variables $x_{i}, s_{k}, x_{l}$ and $w_{j}$ to 1 and the $w$ variables associated with other clauses to 0 . It is clear that the constraints corresponding to the other clauses are trivially satisfied. Likewise, the aggregate constraint $w_{1}+w_{2}+\cdots+w_{m} \geq 1$ is also satisfied. Similar arguments can be made for other forms of $\phi_{j}$.

From Lemma 1 and Lemma 2 and the subsequent discussion, $x_{i}$ and $y_{i}$ can be restricted to the set $\{0,1\}$. Furthermore, the existential player must choose $s_{i}=y_{i}$. Observe that for each $i, r_{i} \leq x_{i}$ and $r_{i} \leq 1-x_{i}$ imply that $r_{i} \leq 0$ (thus satisfying the corresponding constraint in $F$ ). Similarly, for any $i$, the constraints $0 \leq x_{i} \leq 1,0 \leq y_{i} \leq 1,0 \leq s_{i} \leq 1$, $2 \cdot y_{i}-1 \leq s_{i}$, and $s_{i} \leq 2 \cdot y_{i}$ are all satisfied, under this assignment. Hence, $E \rightarrow F$ holds. Since the play was chosen arbitrarily, the same argument applies for all plays, i.e., $\mathbf{X}$ has a winning strategy for $\mathbf{I}$ and $\mathbf{I}$ is feasible.

Theorem (5) allows us to obtain the following two results.

Corollary $\mathbf{3}\left\langle k, \exists, \mathbf{B}^{k+1}\right\rangle$ with $k$ even, is $\Sigma_{\mathbf{k}} \mathbf{P}$-hard.

Proof Let $\boldsymbol{\Phi}$ denote a Q3DNF formula having $k(k$ even) quantifiers $((k-1)$ quantifier alternations) starting with $\exists$, i.e., with a quantifier string of the form $\exists \forall \ldots \exists \forall$. Deciding the feasibility of $\boldsymbol{\Phi}$ is $\Sigma_{\mathbf{k}} \mathbf{P}$-complete. This is because this problem is the complement of the problem $\forall \exists \ldots \forall \exists \phi(\mathbf{x}, \mathbf{y})$, where $\phi(\mathbf{x}, \mathbf{y})$ is a $3 \mathrm{CNF}$ formula, which is $\Sigma_{\mathbf{k}} \mathbf{P}$-complete [22] (the assumption that $k$ is even is essential). By Theorem 5, we can reduce $\boldsymbol{\Phi}$ to a QLI, I. Note that the quantifier string of $\mathbf{I}$ has $k$ quantifier alternations, and that the first and last 
quantifiers are $\exists$. Thus, $\mathbf{I}$ is a $\left\langle k, \exists, \mathbf{B}^{k} \mathbf{R}\right\rangle$ QLI. This can be trivially reduced to a $\left\langle k, \exists, \mathbf{B}^{k+1}\right\rangle$ QLI. Hence, the result follows.

Corollary $4\left\langle k, \forall, \mathbf{B}^{k+1}\right\rangle$ with $k$ odd, is $\Pi_{\mathbf{k}} \mathbf{P}$-hard.

Proof Let $\boldsymbol{\Phi}$ denote a Q3DNF formula having $k$ ( $k$ odd) quantifiers $((k-1)$ quantifier alternations) starting with $\forall$, i.e., with a quantifier string of the form $\forall \exists \ldots \exists \forall$. Deciding the feasibility $\boldsymbol{\Phi}$ belongs to is $\Pi_{\mathbf{k}} \mathbf{P}$-complete. This is because this problem is the complement of $\exists \forall \ldots \exists \phi(\mathbf{x}, \mathbf{y})$, where $\phi(\mathbf{x}, \mathbf{y})$ is a $3 \mathrm{CNF}$ formula, which is $\Sigma_{\mathbf{k}} \mathbf{P}$-complete [22] (the assumption that $k$ is odd is essential). By Theorem 5, we can reduce $\boldsymbol{\Phi}$ to a QLI, I. Note that the quantifier string of $\mathbf{I}$ has $k$ quantifier alternations, and that the last quantifiers is $\exists$. Thus, I is a $\left\langle k, \forall, \mathbf{B}^{k} \mathbf{R}\right\rangle$ QLI. This can be trivially reduced to a $\left\langle k, \forall, \mathbf{B}^{k+1}\right\rangle$ QLI. Hence, the result follows.

Theorem $6\left\langle k, \exists, \mathbf{B}^{k+1}\right\rangle$ is $\mathbf{\Sigma}_{\mathbf{k}} \mathbf{P}$-complete, $\left\langle k, \forall, \mathbf{B}^{k+1}\right\rangle$ is $\Pi_{\mathbf{k}} \mathbf{P}$-complete.

Proof Given Theorems 3-4 and Corollaries 3-4, it suffices to show that each of these problems is also contained within the corresponding complexity class of the polynomial hierarchy. Let $\mathbf{L}$ denote the problem of deciding an arbitrary boolean combination of linear constraints under a quantifier string with a limited number of alternations. Sontag [31] showed that problem $\mathbf{L}$ can be solved by an alternating algorithm in which the guesses made by both the $\forall$ player and the $\exists$ player are rational and at most polynomial in the size of the input. QLIs with a finite number of quantifier alternations are clearly sub-problems of $\mathbf{L}$, since they can be rewritten as a quantified disjunction of the RHS constraints and the negation of the LHS constraints. It follows that the feasibility of QLIs with a finite number of alternations is preserved, even if every variable is restricted to values that are polynomially-sized with respect to the input. Consider the problem $\left\langle k, \exists, \mathbf{B}^{k+1}\right\rangle$. After $k$ rounds in which the $\exists$ player and $\forall$ player alternate and guess polynomially-sized values, the QLI reduces to either $\langle 0, \exists, \mathbf{B}\rangle$ or $\langle 0, \forall, \mathbf{B}\rangle$ (depending on whether $k$ is even or odd respectively), both of which are in $\mathbf{P}$ [14]. Thus, $\left\langle k, \exists, \mathbf{B}^{k+1}\right\rangle$ is in $\Sigma_{\mathbf{k}} \mathbf{P}$. Likewise, $\left\langle k, \forall, \mathbf{B}^{k+1}\right\rangle$ is in $\Pi_{\mathbf{k}} \mathbf{P}$.

For example, QLIs of the form $\langle 3, \forall, \mathbf{B B B B}\rangle$ are in $\Pi_{3} \mathbf{P}$.

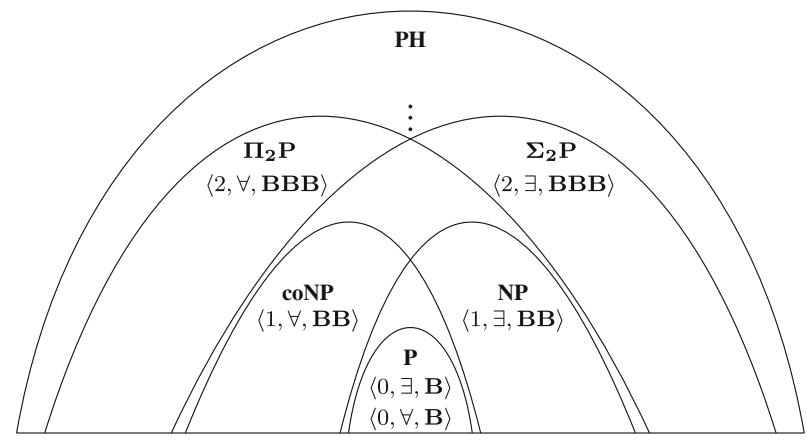

Fig. 2 QLI and the Polynomial Hierarchy 
The various forms of QLI cover the polynomial hierarchy as shown in Fig. 2.

We make the following observations on the basis of the theorems derived above:

(i) In case of QLPs, if the innermost variable is universally quantified, it can be removed using quantifier elimination techniques, in polynomial time. In case of QLIs, the complexity class to which the problem belongs, does in fact depend upon whether the innermost variable is existentially or universally quantified (see Fig. 2).

(ii) There exists a class of QLI that is complete for each class in the PH. This is not true, if the underlying formula is in CNF form. For instance, there is no QCNF formula, which is complete for the class coNP.

\section{Other complexity results}

In this section, we establish the computational complexities of various classes of QLI with one quantifier alternation that were left open in [14].

Lemma $3\langle 1, \forall, \mathbf{R B}\rangle$, i.e., deciding whether $\forall \mathbf{y} \exists \mathbf{x}[\mathbf{M} \cdot \mathbf{x} \leq \mathbf{n} \rightarrow \mathbf{A} \cdot \mathbf{x}+\mathbf{B} \cdot \mathbf{y} \leq \mathbf{c}]$ holds, is in $\mathbf{P}$.

Proof We will focus on the following two cases:

1. There exists a point $\mathbf{z}$, such that $\mathbf{M} \cdot \mathbf{z} \not \leq \mathbf{n}$ - It follows that $\forall \mathbf{x}(\mathbf{M} \cdot \mathbf{x} \leq \mathbf{n})$ does not hold. In this case, the QLI is trivially satisfied, since the existential player can guess $\mathbf{z}$ and cause the left hand side of the implication to be falsified.

2. There is no point $\mathbf{z}$ such that $\mathbf{M} \cdot \mathbf{z} \not \leq \mathbf{n}$ - In this case, the QLI reduces to $\forall \mathbf{y} \exists \mathbf{x} \mathbf{A} \cdot \mathbf{x}+\mathbf{B}$. $\mathbf{y} \leq \mathbf{c}$, which is a UQLP and hence can be decided in polynomial time by Theorem 2 .

The decision problem for formula $\exists \mathbf{x} \forall \mathbf{y}[\mathbf{A} \cdot \mathbf{x}+\mathbf{B} \cdot \mathbf{y} \leq \mathbf{c} \rightarrow \mathbf{M} \cdot \mathbf{y} \leq \mathbf{n}]$ is NPcomplete [14]. However, if there are no universally quantified variables on the RHS, the problem becomes tractable.

Lemma $4\langle 1, \exists, \mathbf{B L}\rangle$, i.e., deciding whether $\exists \mathbf{x} \forall \mathbf{y}[\mathbf{A} \cdot \mathbf{x}+\mathbf{B} \cdot \mathbf{y} \leq \mathbf{c} \rightarrow \mathbf{M} \cdot \mathbf{x} \leq \mathbf{n}]$ holds, is in $\mathbf{P}$.

Proof First, we check whether $\exists \mathbf{x} \mathbf{M} \cdot \mathbf{x} \leq \mathbf{n}$ holds, which can be done in polynomial time [20]. If $\exists \mathbf{x} \mathbf{M} \cdot \mathbf{x} \leq \mathbf{n}$ holds, then $\exists \mathbf{x} \forall \mathbf{y}[\mathbf{A} \cdot \mathbf{x}+\mathbf{B} \cdot \mathbf{y} \leq \mathbf{c} \rightarrow \mathbf{M} \cdot \mathbf{x} \leq \mathbf{n}]$ trivially holds. If $\exists \mathbf{x} \mathbf{M} \cdot \mathbf{x} \leq \mathbf{n}$ does not hold, then the only way in which $\exists \mathbf{x} \forall \mathbf{y}[\mathbf{A} \cdot \mathbf{x}+\mathbf{B} \cdot \mathbf{y} \leq \mathbf{c} \rightarrow \mathbf{M} \cdot \mathbf{x} \leq \mathbf{n}]$ can hold is if $\exists \mathbf{x} \forall \mathbf{y} \mathbf{A} \cdot \mathbf{x}+\mathbf{B} \cdot \mathbf{y} \leq \mathbf{c}$ does not hold. However, the latter formula is a UQLP and hence it can be checked to hold in polynomial time by Theorem 2 .

Let us turn our attention to the class $\langle 1, \forall, \mathbf{B R}\rangle$ and its super-class $\langle 1, \forall, \mathbf{B B}\rangle$. Both these classes were shown to be coNP-hard in [14]. Note that these classes are also in coNP by Theorem 6.

Lemma 3 and Lemma 4 settle some open problems on the computational complexities of QLIs with one quantifier alternation. A complete representation of all classes with one quantifier alternation QLIs is given in Fig. 3 (starting with an existential quantifier) and Fig. 4 (starting with a universal quantifier). 


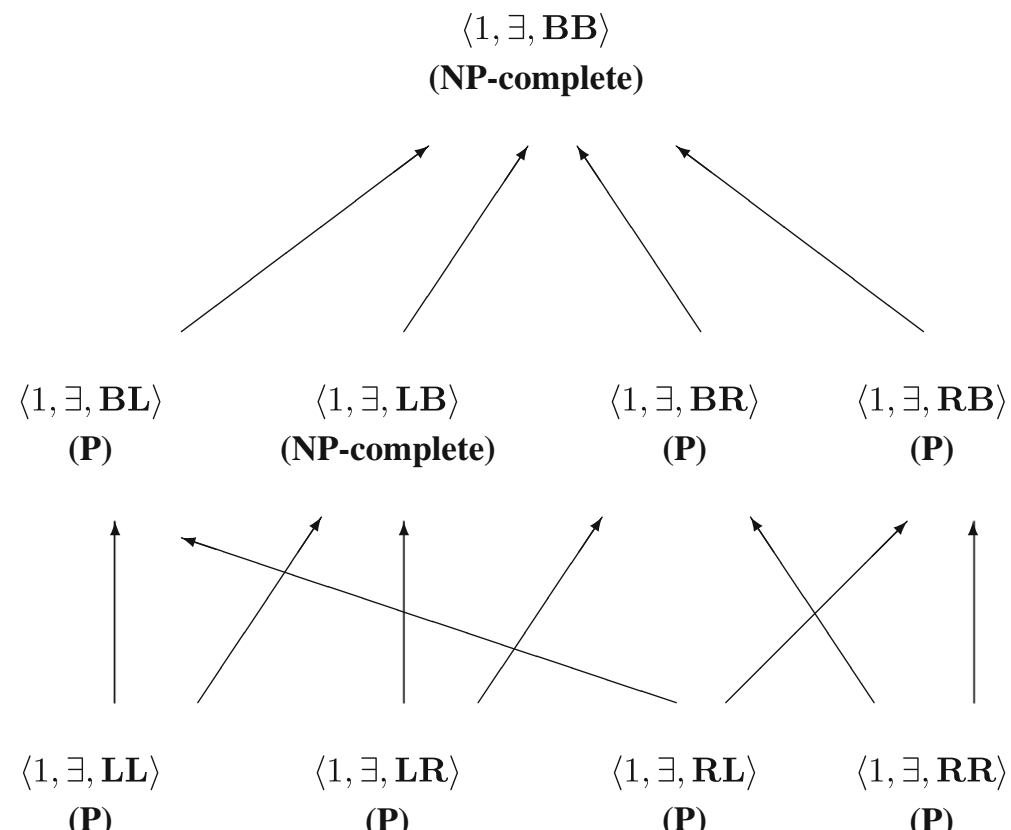

Fig. 3 Complexity of $\exists \forall$ classes of QLI. Arrows denote inclusions

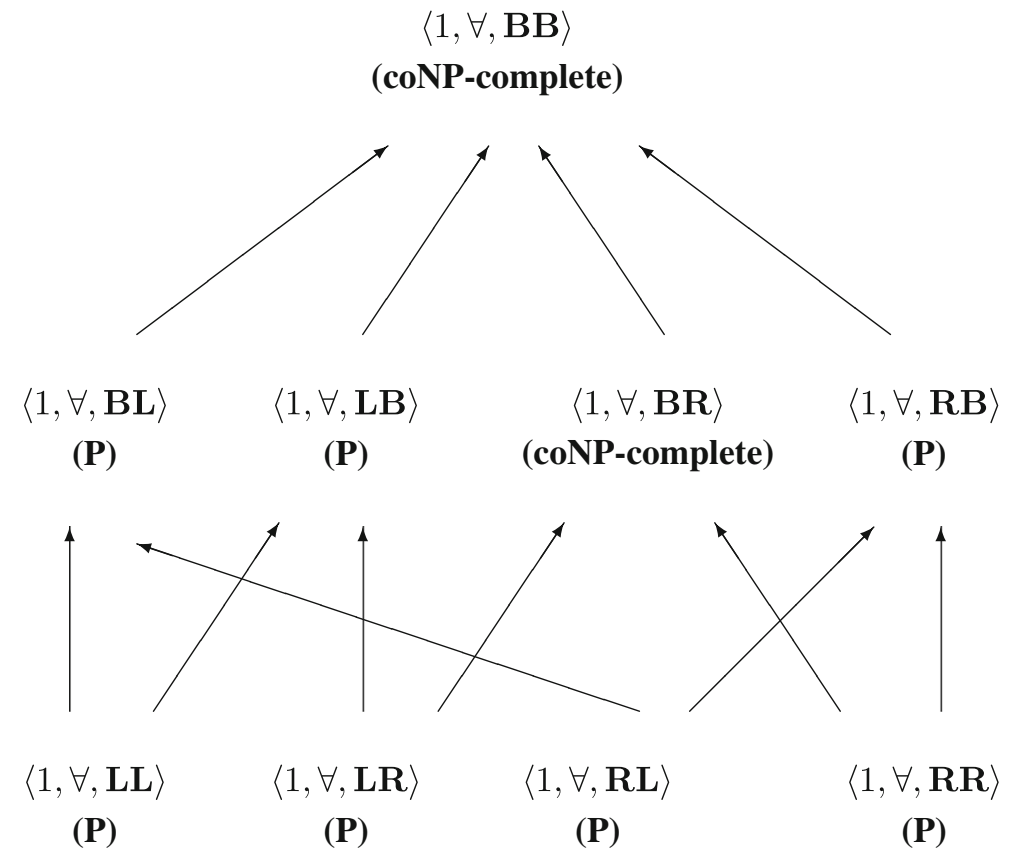

Fig. 4 Complexity of $\forall \exists$ classes of QLI. Arrows denote inclusions 


\section{Conclusion}

In this paper, we established the computational complexities of various fragments of the theory of linear arithmetic. More specifically, we examined several variants of QLP and QLI that arise when the universally quantified variables are partially bounded or unbounded and showed that all these variants are in $\mathbf{P}$. Furthermore, for QLIs, we proved that $\left\langle k, \exists, \mathbf{B}^{k+1}\right\rangle$ is $\Sigma_{\mathbf{k}} \mathbf{P}$-complete and $\left\langle k, \forall, \mathbf{B}^{k+1}\right\rangle$ is $\Pi_{\mathbf{k}} \mathbf{P}$-complete. Hence, we showed that any class of PH can be represented by some instantiation to the QLI framework, i.e., using only continuous variables, thus providing a continuous analogue to the way QBFs cover the PH. In particular, our work mirrors the work in [32], which established the connection between the classes of PH and the quantifier alternations in Quantified Boolean Formulas. Our results are surprising, since the variables in the fragments that we consider are continuous, as opposed to discrete. Moreover, we answered several open questions on the computational complexities of classes of QLI with one quantifier alternation, thus completing the map of the computational complexities of all such classes of QLI (see Figs. 3 and 4).

From our perspective, the following problems are worth pursuing:

1. What is the complexity of Quantified Linear Programming? As discussed previously, we have shown in previous research that the problem is in PSPACE and coNP-hard. Note that our results are similar to the results obtained in [6] for Quantified Horn formulas.

2. What is the complexity of Quantified Linear Implication? It is known that the decidability of generic QLIs with unbounded alternations is PSPACE-hard [14]. However, completeness is not yet shown. Note that the known decision methods for TLA with an arbitrary number of quantifier alternations run in double exponential time $[15,31]$.

3. In deriving our complexity results, we mostly instantiated the $T$ parameter in $\langle A, Q, T\rangle$ to $\mathbf{B}$. It remains to be seen if and how the complexity results change when $T$ is assigned $\mathbf{L}$ or $\mathbf{R}$. At this point, it is important to note that the assignments $\mathbf{L}$ and $\mathbf{R}$ are not symmetric from the perspective of computational complexity [14].

\section{Compliance with Ethical Standards}

Conflict of Interest The authors declare that they have no conflict of interest.

Funding This research was supported in part by the National Science Foundation through Award CCF1305054 and the Air Force Office of Scientific Research through Award FA9550-12-1-0199. This research has been co-financed by the European Union (European Social Fund - ESF) and Greek national funds through the Operational Program "Education and Lifelong Learning" of the National Strategic Reference Framework (NSRF) - Research Funding Program: Thales. Investing in knowledge society through the European Social Fund (MIS: 380232).

\section{Appendix}

\section{A Axioms}

We now provide the axioms of each theory mentioned in the paper. 


\section{A.1 Theory of linear arithmetic}

The Theory of Linear Arithmetic, also known as the Theory of Rationals, is a set of first order axioms which define the properties of rational numbers.

The signature of this theory is:

$$
\Sigma_{\mathbb{Q}}=\{0,1,+,-,=, \geq\}
$$

The axioms of this theory are as follows:

1. $\forall x \forall y \quad x \geq y \wedge y \geq x \rightarrow x=y$.

2. $\forall x \forall y \forall z \quad x \geq y \wedge y \geq z \rightarrow x \geq z$.

3. $\forall x \forall y \quad x \geq y \vee y \geq x$.

4. $\forall x \forall y \forall z(x+y)+z=x+(y+z)$.

5. $\forall x \quad x+0=x$.

6. $\forall x \quad x+(-x)=0$.

7. $\forall x \forall y \quad x+y=y+x$.

8. $\forall x \forall y \forall z \quad x \geq y \rightarrow x+z \geq y+z$.

9. $\forall x \forall n \in \mathbb{Z}^{+} n \cdot x=0 \rightarrow x=0$.

10. $\forall x \forall n \in \mathbb{Z}^{+} \exists y \quad x=n \cdot y$.

\section{A.2 Theory of real closed fields}

The Theory of Real Closed Fields is a set of axioms which define the properties of real numbers.

The signature of this theory is:

$$
\Sigma_{\mathbb{R}}=\{0,1,+,-, \cdot,=, \geq\} .
$$

The first order axioms of this theory are as follows:

1. $\forall x \forall y \quad x \geq y \wedge y \geq x \rightarrow x=y$.

2. $\forall x \forall y \forall z \quad x \geq y \wedge y \geq z \rightarrow x \geq z$.

3. $\forall x \forall y \quad x \geq y \vee y \geq x$.

4. $\forall x \forall y \forall z(x+y)+z=x+(y+z)$.

5. $\forall x \quad x+0=x$.

6. $\forall x \quad x+(-x)=0$.

7. $\forall x \forall y \quad x+y=y+x$.

8. $\forall x \forall y \forall z \quad x \geq y \rightarrow x+z \geq y+z$.

9. $\forall x \forall y \forall z(x \cdot y) \cdot z=x \cdot(y \cdot z)$.

10. $\forall x \quad x \cdot 1=x$.

11. $\forall x \quad x \neq 0 \rightarrow \exists y \quad x \cdot y=1$.

12. $\forall x \forall y \quad x \cdot y=y \cdot x$.

13. $\forall x \forall y \quad x \geq 0 \wedge y \geq 0 \rightarrow x \cdot y \geq 0$.

14. $\forall x \forall y \forall z \quad x \cdot(y+z)=x \cdot y+x \cdot z$.

15. $0 \neq 1$.

16. $\forall x \exists y \quad x=y^{2} \vee-x=y^{2}$.

17. $\forall \mathbf{x} \forall n \in \mathbb{Z}^{+} \exists y n \bmod 2 \equiv 1 \rightarrow y^{n}+x_{1} \cdot y^{n-1}+\ldots+x_{n-1} \cdot y+x_{n}=0$. 
However these fail to fully describe the real numbers. To do this, we need the following second order axiom:

$$
\forall S \subseteq \mathbb{R} \exists x_{1} \exists y \forall x_{2} \in S \forall x_{3}>y \exists x_{4} \in S x_{1} \leq x_{2} \rightarrow\left(y \leq x_{2} \wedge x_{4}<x_{3}\right) .
$$

This axiom represents the Dedekind completeness of real numbers. Namely that every set $S$ that has a lower bound $x_{1}$ has a greatest lower bound $y$.

\section{B Game semantics}

We provide 2-person game semantics for QLP, QLI, and Q3DNF.

\section{B.1 QLP semantics}

We interpret QLP decidability as a 2-person game. Such a game includes an existential player $\mathbf{X}$, who chooses values for the existentially quantified variables, and a universal player $\mathbf{Y}$, who chooses values for the universally quantified variables. Our analysis focuses on QLPs in general form [33], but also holds for the partially bounded and unbounded variants, discussed in this paper.

Consider the generic form of QLP (i.e., QLP (3)) and assume, without loss of generality, that $\mathbf{x}_{1}$ and $\mathbf{y}_{n}$ are not empty (dummy variables can be added, if necessary). The initial board configuration of the game is:

$$
\mathbf{A} \cdot \mathbf{x}+\mathbf{N} \cdot \mathbf{y} \leq \mathbf{b}
$$

The game is played in a sequence of $2 \cdot n$ rounds. Let $i=1, \ldots, n$. In round $(2 \cdot i-1)$, $\mathbf{X}$ makes his $i^{t h}$ move (by choosing values for the variables in the set $\mathbf{x}_{i}$ ). Then, $\mathbf{Y}$ makes his $i^{t h}$ move (by choosing values for the variables in the set $\mathbf{y}_{i}$ ) in round $2 \cdot i$. Hence, $\mathbf{X}$ and $\mathbf{Y}$ make their moves by selecting values for their respective variable sets. The moves are strictly alternating: $\mathbf{X}$ makes his $i^{\text {th }}$ move, which is followed by $\mathbf{Y}$ 's $i^{\text {th }}$ move, after which $\mathbf{X}$ makes his $(i+1)^{t h}$ move and so on. After all the moves have been made in the order specified by the quantifier string, if $\mathbf{A} \cdot \mathbf{x}+\mathbf{N} \cdot \mathbf{y} \leq \mathbf{b}$ holds, we say that $\mathbf{X}$ wins the game; otherwise, we say that $\mathbf{Y}$ wins the game.

\section{B.2 QLI semantics}

We now interpret a QLI as a 2-person game. As with the QLP semantics discussed above, such a game includes an existential player $\mathbf{X}$, who chooses values for the existentially quantified variables, and a universal player $\mathbf{Y}$, who chooses values for the universally quantified variables. Again, our analysis focuses on QLIs of generic form but also holds for the partially bounded and unbounded variants.

Consider the generic form of QLI (i.e., QLI (7)), and assume, without loss of generality, that $\mathbf{x}_{1}$ and $\mathbf{y}_{n}$ are not empty (dummy variables can be added, if necessary). The initial board configuration of the game is:

$$
[\mathbf{A} \cdot \mathbf{x}+\mathbf{N} \cdot \mathbf{y} \leq \mathbf{b} \rightarrow \mathbf{C} \cdot \mathbf{x}+\mathbf{M} \cdot \mathbf{y} \leq \mathbf{d}]
$$

The game is played in a sequence of $2 \cdot n$ rounds. Let $i=1, \ldots, n$. In round $(2 \cdot i-1)$, $\mathbf{X}$ makes his $i^{t h}$ move (by choosing values for the variables in the set $\mathbf{x}_{i}$ ). Then, $\mathbf{Y}$ makes his $i^{\text {th }}$ move (by choosing values for the variables in the set $\mathbf{y}_{i}$ ) in round $2 \cdot i$. Hence, $\mathbf{X}$ and 
Y make their moves by selecting values for their respective variable sets. The moves are strictly alternating: $\mathbf{X}$ makes his $i^{\text {th }}$ move, which is followed by $\mathbf{Y}$ 's $i^{\text {th }}$ move, after which $\mathbf{X}$ will makes $(i+1)^{t h}$ move and so on. Assume that all the moves have been made by both players in the order specified by the quantifier string. Let $\mathbf{u}=\mathbf{A} \cdot \mathbf{x}+\mathbf{N} \cdot \mathbf{y}$ and let $\mathbf{v}=\mathbf{C} \cdot \mathbf{x}+\mathbf{M} \cdot \mathbf{y}$. If $\mathbf{u} \leq \mathbf{b}$ and $\mathbf{v} \not \mathbf{d}$, we say that $\mathbf{Y}$ wins the game. Otherwise, $\mathbf{X}$ wins the game.

\section{B.3 Q3DNF semantics}

We now discuss game semantics for Q3DNF. Again, the existential and the universal player make moves according to the quantifier string. The existential player wins a (disjunctive) clause, if all the literals in the clause are set to true. Conversely the universal player wins the clause, if at least one literal is set to false. The existential player wins the game, if he wins at least one clause in the disjunction, thus causing the expression to evaluate to true. The universal player wins the game, if he wins every term in the disjunction, thus causing the expression to evaluate to false. This can be considered as a reversal of the players' objectives in an instance of Q3SAT, where the existential player needs to win all the conjunctive clauses, and the universal player only needs to win a single clause. This occurs because the underlying boolean formula of a Q3DNF instance is in DNF, while the underlying boolean formula of a Q3SAT instance is in CNF.

\section{References}

1. Basu, S., Pollack, R., Roy, M.-F.: Existential theory of the reals, volume 10 of algorithms and computation in mathematics. Springer, Berlin (2006)

2. Berman, L.: The complexity of logical theories. Theor. Comput. Sci. 11, 71-77 (1980)

3. Bialas, W.F., Karwan, M.H.: Two-level linear programming. Manag. Sci. 30(8), 1004-1020 (1984)

4. Bradley, A.R., Manna, Z. The calculus of computation - decision procedures with applications to verification, first edition. Springer, Berlin (2007)

5. Brown, C.W.: QEPCAD B: A Program for computing with semi-algebraic sets using CADs. ACM SIGSAM Bull. 37(4), 97-108 (2003)

6. Büning, H.K., Karpinski, M., Flögel, A.: Resolution for quantified boolean formulas. Inf. Comput. 117(1), 12-18 (1995)

7. Canny, J.: Some algebraic and geometric computations in PSPACE. Proceedings of the Twentieth Annual ACM Symposium on Theory of Computing, 460-467 (1988)

8. Choi, S., Agrawala, A.: Dynamic dispatching of cyclic real-time tasks with relative timing constraints. Real-Time Syst. 19(1), 5-40 (2000)

9. Collins, G.E., Hong, H.: Partial cylindrical algebraic decomposition for quantifier elimination. J. Symb. Comput. 12(3), 299-328 (1991)

10. Davenport, J.H., Heintz, J.: Real quantifier elimination is doubly exponential. J. Symb. Comput. 5(1-2), 29-35 (1988)

11. Dolzmann, A., Sturm, T.: REDLOG: Computer algebra meets computer logic. ACM SIGSAM Bull. 31(2), 2-9 (1997)

12. Dolzmann, A., Sturm, T., Weispfenning, V.: A new approach for automatic theorem proving in real geometry. J. Autom. Reason. 21(3), 357-380 (1998)

13. Dolzmann, A., Sturm, T., Weispfenning, V.: Real quantifier elimination in practice. In: Matzat, B.H., et al. (eds.) Algorithmic Algebra and Number Theory, pages 221-248. Springer (1998)

14. Eirinakis, P., Ruggieri, S., Subramani, K., Wojciechowski, P.: On quantified linear implications. Ann. Math. Artif. Intell. 71(4), 301-325 (2014)

15. Ferrante, J., Rackoff, C.: A decision procedure for the first order theory of real addition with order. SIAM J. Comput. 4(1), 69-76 (1975) 
16. Gerber, R., Pugh, W., Saksena, M.: Parametric dispatching of hard real-time tasks. IEEE Trans. Comput. 44(3), 471-479 (1995)

17. Hall, R.J.: Specification, validation, and synthesis of email agent controllers: A case study in function rich reactive system design. Autom. Softw. Eng. 9(3), 233-261 (2002)

18. Harel, D.: A grand challenge for computing: Towards full reactive modeling of a multi-cellular animal. Verification, Model Checking, Abstr. Interpretation 2937, 39-60 (2004)

19. Kam, N., Cohen, I.R., Harel, D.: The immune system as a reactive system: modeling t cell activation with statecharts. In: Proceedings of the 2001 IEEE Symposia on Human-Centric Computing Languages and Environments, pp. 15-22 (2001)

20. Khachiyan, L.G.: A polynomial algorithm in linear programming. Doklady Akademiia Nauk SSSR 224, 1093-1096 (1979). English Translation: Soviet Mathematics Doklady, Volume 20, pp. 1093-1096

21. Koo, T.J., Sinopoli, B., Sangiovanni-Vincentelli, A., Sastry, S.: A formal approach to reactive system design: unmanned aerial vehicle flight management system design example. In: Proceedings of the 1999 IEEE International Symposium on Computer Aided Control System Design, pp. 522-527 (1999)

22. Christos, H.: Papadimitriou computational complexity Addison-Wesley (1994)

23. Pfitzmann, B., Waidner, M.: Composition and integrity preservation of secure reactive systems. In: Proceedings of the 2000 ACM Conference on Computer Communications Security, pp. 245-254 (2000)

24. Pfitzmann, B., Waidner, M.: A model for asynchronous reactive systems and its application to secure message transmission. In: Proceedings of the 2001 IEEE Symposium on Security and Privacy, pp. 184 200 (2001)

25. Ratschan, S.: Efficient solving of quantified inequality constraints over the real numbers. ACM Trans. Comput. Log. 7(4), 723-748 (2006)

26. Ratschan, S.: RSOLVER. http://rsolver.sourceforge.net (2013)

27. Schaefer, M., Umans, C.: Completeness in the polynomial-time hierarchy A compendium. SIGACT News 33(3), 32-49 (2002)

28. Schaefer, M., Umans, C.: Completeness in the polynomial-time hierarchy Part II. SIGACT News 33(4), 22-36 (2002)

29. Schrijver, A.: Theory of linear and integer programming. John Wiley and Sons, New York (1987)

30. Shor, P.W.: Stretchability of pseudolines is NP-hard. DIMACS 4, 531-534 (1991)

31. Sontag, E.D.: Real addition and the polynomial hierarchy. Inf. Process. Lett. 20(3), 115-120 (1985)

32. Stockmeyer, L.J.: The polynomial-time hierarchy. Theor. Comput. Sci. 3, 1-22 (1977)

33. Subramani, K.: On a decision procedure for quantified linear programs. Ann. Math. Artif. Intell. 51(1), 55-77 (2007)

34. Tarski, A.: A decision method for elementary algebra and geometry. Technical report r-109 rand corporation (1948)

35. Weispfenning, V.: The complexity of linear problems in fields. J. Symb. Comput. 4(1-2), 3-27 (1988) 